Chemometrics and Intelligent Laboratory Systems, 10 (1991) 199-210

Elsevier Science Publishers B.V., Amsterdam

\title{
Stochastic aspects of turbulent combustion processes
}

\author{
G.M. Faeth *, M.E. Kounalakis and Y.R. Sivathanu \\ Department of Aerospace Engineering, The University of Michigan, Ann Arbor, MI (U.S.A.)
}

(Received 8 November 1989; accepted 4 January 1990)

\begin{abstract}
Faeth, G.M., Kounalakis, M.E. and Sivathanu, Y.R., 1991. Stochastic aspects of turbulent combustion processes. Chemometrics and Intelligent Laboratory Systems, 10: 199-210.

Methods of using stochastic simulations to treat nonlinear interactions in turbulent combustion processes are described emphasizing the use of statistical time-series techniques to analyze the turbulence-radiation interactions of nonpremixed flames. Three aspects of the problem are considered, as follows: the statistics of scalar properties in turbulent flames, the formulation of algorithms to simulate flame radiation based on flame statistics, and evaluation of the methodology using recent measurements for nonluminous flames. It is shown that the process becomes tractable through the laminar flamelet approximation whereby all scalar properties are taken to be solely functions of a conserved scalar like the mixture fraction. Thus, the simulations are designed to generate realizations of mixture fractions along radiation paths with the radiation properties of each realization found using a narrow-bond radiation model. An autoregressive process that reproduces probability density functions and spatial and temporal correlations of mixture fractions was found to yield reasonably good predictions of the statistical properties of spectral radiation intensities measured for turbulent carbon monoxide and hydrogen jet flames burning in still air. Although the approach appears to be promising, additional development is needed in order to treat some of the unique statistical features of turbulence that are not encountered during conventional use of statistical time-series techniques.
\end{abstract}

\section{INTRODUCTION}

Stochastic simulations are promising for treating a variety of nonlinear interactions in turbulent flows. Recent studies along these lines include the turbulent dispersion of particles and bubbles [1-5], the motion and transport of drops in evaporating and combusting sprays $[6,7]$, and the turbulenceradiation interactions of nonpremixed flames [813]. The objective of the present paper is to describe the application of this methodology to processes encountered in turbulent combusting flows. In order to control the scope, the discussion will focus on turbulence-radiation interactions of nonpremixed (diffusion) flames, since this problem involves the most significant features of stochastic simulations of turbulent combustion processes.

Initially, methods of simulating turbulent processes were relatively ad hoc [1,2]; however, more systematic techniques currently are being emphasized. This includes full stochastic simulation of the turbulent field, along the lines of Kraichnan [14], to study the turbulent dispersion 
of particles in an isotropic turbulent field [15], and adapting statistical time-series techniques, analogous to methods described by Box and Jenkins [15], for problems of turbulent dispersion of particles [3,5] and turbulence-radiation interactions [13]. The present discussion will be limited to statistical time-series techniques since they have modest computational requirements and provide reasonable flexibility for treating a variety of practical turbulent flows.

The main reason for interest in turbulenceradiation interactions is that radiation levels of turbulent flames are generally higher (often 2-3 times higher) than estimates based on mean scalar properties within the flames [8-12]. The bias of mean radiation levels is caused by nonlinear relationships between scalar and radiation properties in flames. This precludes averaging scalar properties first and then computing radiation properties; instead, the radiation properties of realizations of the scalar field must be found first and then averaged. Properties other than mean radiation levels are also of interest; for example, fire and flame detectors often use the temporal properties of flame radiation fluctuations to distinguish flames from background radiation. Furthermore, maximum (rather than average) flame radiation levels provide the most conservative estimate of flame radiation properties for fire safety considerations. Finally, studying the temporal properties of radiation fluctuations (moments, probability density functions, and temporal power spectral densities) provides information to better understand turbulence-radiation interactions, analogous to the information provided by the temporal properties of velocity and concentration fluctuations to better understand turbulent mixing. Thus, the general problem of turbulence-radiation interactions involves both the mean and fluctuating radiation properties of turbulent flames [11,12].

Statistical time-series simulations of the radiation properties of turbulent flames are based on simulation of scalar properties within the flames. Therefore, the paper begins with a description of the statistics of scalar properties in turbulent flames. The formulation of typical stochastic simulations is then considered. The paper concludes with evaluation of the methodology using measurements from turbulent hydrogen and carbon monoxide jet flames burning in still air.

\section{SCALAR PROPERTIES OF DIFFUSION FLAMES}

\section{Scalar property correlations}

Assuming equal exchange coefficients of all species and heat, negligible effects of potential and kinetic energies and radiation, and reaction occurring at an infinitely-thin flame sheet, Burke and Schumann [16] showed that scalar properties in laminar nonpremixed flames were functions (called state relationships) of any one of a number of conserved scalars. Although the formal requirements are rather restrictive, state relationships have been found for many laminar flame systems and are widely used for analysis of flame structure and radiation properties. The use of state relationships has also been extended to turbulent nonpremixed flames, since they generally can be approximated as wrinkled laminar flames. The use of state relationships for turbulent nonpremixed flames has come to be called the conserved-scalar formalism under the laminar flamelet approximation $[17,18]$.

Typical state relationships are illustrated in Fig. 1. This involves measurements of the concentrations of major gas species and temperature, $T$, for radial traverses at various heights, $x$, above a burner having diameter, $d$, as well as axial traverses, within laminar nonpremixed carbon monoxide/air flames having various burner Reynolds numbers, $R e$. In this case, the conserved scalar is the local fuel-equivalence ratio (the mass fraction of fuel elements irrespective of species divided by the stoichiometric mass fraction of fuel elements). Predictions based on the assumption of local thermodynamic equilibrium for an adiabatic flame, using the Gordon and McBride [19] algorithm, are also shown on the figure. Aside from temperature (where radiative heat losses and errors of uncorrected temperature measurements are a factor) the measured state relationships are seen to be in excellent agreement with equilibrium predictions. Thus, the tendency of reactive systems to approach equilibrium provides a physical justifica- 


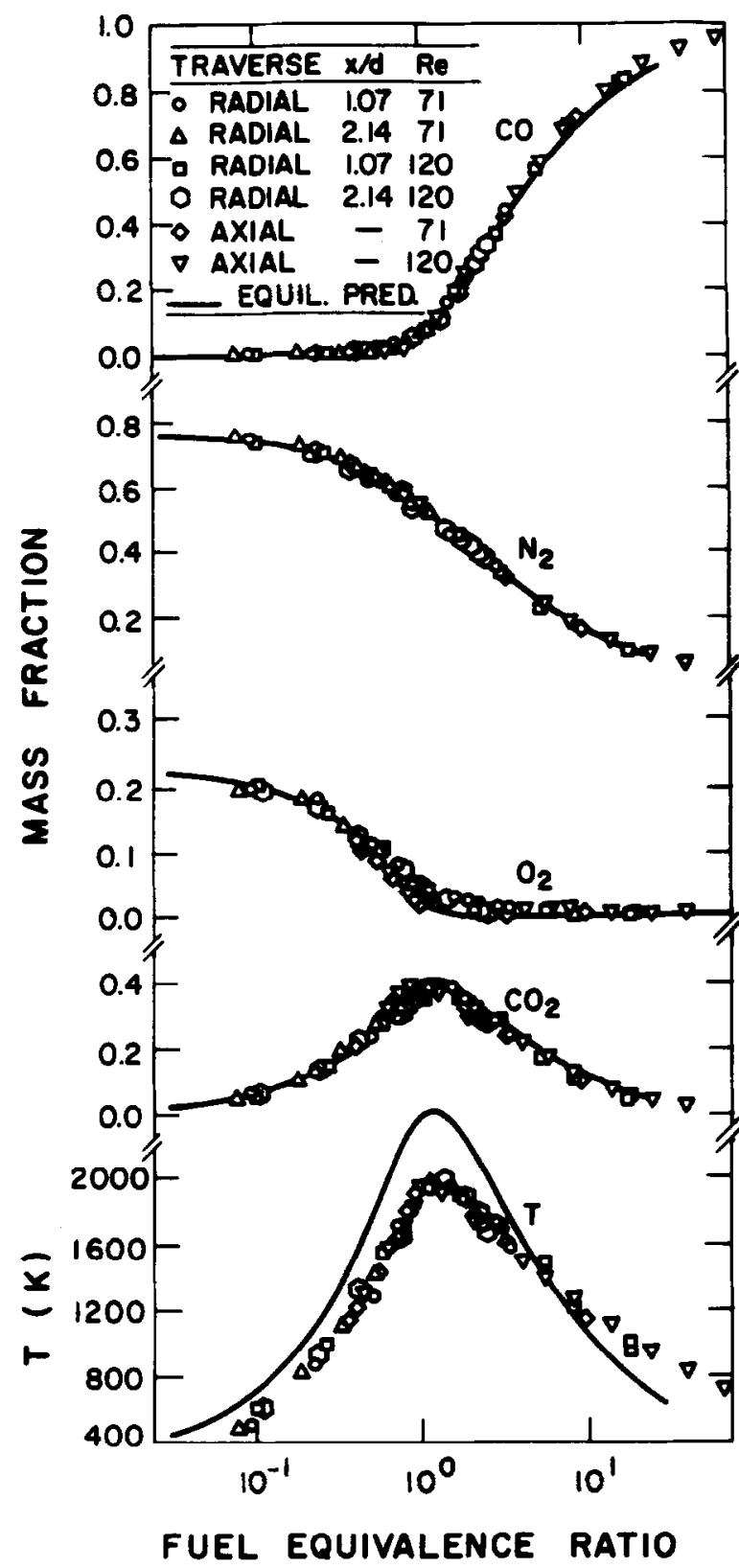

Fig. 1. State relationships for carbon monoxide/air diffusion flames. From Gore et al. [8].

tion for the laminar flamelet approximation in this instance.

State relationships for the concentrations of major gas species and temperature, adequate for estimates of structure and radiation properties, have been found from measurements in laminar flames for a variety of fuels burning in air: hydrogen $[9,17]$, methane $[18,20,21]$, propane $[22], n$ heptane [17,23], acetylene [11] and ethylene [10]. Hydrocarbons exhibit significant departures from local thermodynamic equilibrium at fuel-rich conditions due to effects of finite-rate chemistry associated with soot processes; however, these departures are still relatively universal so that adequate state relationships are still found except near points of flame attachment. Finally, generalized state relationships have been found for hydrocarbon/air flames so that tedious measurements to find state relationships for specific fuels can be avoided [22].

Application of the conserved-scalar formalism and the laminar flamelet approximation to find the structure of turbulent flames has been reasonably successful for virtually all the materials for which state relationships are available [8$13,17,24,25]$. Recent studies also suggest that state relationships for soot volume fractions, an important property for estimates of continuum radiation from soot, exist in turbulent flames having sufficiently long residence times $[26,27]$. This implies that scalar properties needed to estimate radiation are strongly correlated through their state relationships and can be simulated by simulating a conserved-scalar alone.

\section{Mixture fraction statistics}

Mixture fraction, $f$, defined as the fraction of elemental mass that originated from the fuel, is the conserved scalar most commonly used to find the scalar structure of turbulent nonpremixed flames. Turbulence models under the conservedscalar formalism are designed to provide estimates of the mean value and variance of mixture fractions $[17,28]$. Methods used to estimate the other statistical properties needed to simulate mixture fraction distributions along radiation paths probability density functions and correlations will be considered in the following.

A fuel burning in air involves instantaneous properties at any point that can be pure air, pure fuel or some mixture of the two with scalar properties given by the state relationships. Several 
probability density functions (PDFs) of mixture fraction, $P(f)$, have been proposed to accommodate these possibilities but the clipped-Gaussian PDF has received the most attention [28]. This involves a Gaussian function defined in range $0<f<1$ with the tails of the distribution replaced by Dirac delta functions at $f=0$ and 1 that have weights equal to the probability of $f<0$ and $f>1$ for the original Gaussian distribution, respectively. Thus, the air intermittency of the flame at any point, defined as the fraction of time spent in ambient air, is given by the weighted Dirac delta function at $f=0$.

Recent measurements in noncombusting and combusting turbulent flows suggest that the clipped-Gaussian PDF of mixture fraction is reasonable $[29,30]$. Some typical results are illustrated in Fig. 2 for turbulent carbon monoxide jet flames burning in still air. The measurements in the figure are fitted with clipped-Gaussian PDFs having the same mean values and variances. Results are shown for various radial positions, $r$, before and after the flame tip $(x / d=30$ and 50). The air intermittency spike is prominent for these conditions but the fuel intermittency spike can only be seen in the fitted PDFs near the axis at $x / d=30$. The main deficiency of the clipped-Gaussian fits is that they fail to represent the broadened air intermittency spike caused by direct mixing between turbulent fluid and air near the edge of the flow (the air superlayer). A PDF having additional moments is needed to correct this problem; however, the complication of finding addition moments has not been pursued pending evaluation of the performance of the two-moment PDF. Notably, the functions used for mixture fraction PDFs normally do not have a strong effect upon predictions of scalar properties in turbulent flames [28].

Correlations of mixture fraction fluctuations, $f^{\prime}$, have been measured for turbulent jet-like flows for both noncombusting [31,32] and combusting [30] conditions. Some typical spatial correlations are illustrated in Fig. 3 for a carbon monoxide jet diffusion flame burning in still air. These results involve two-point spatial correlations of mixture fraction fluctuations for horizontal radial paths through the flame axis at positions before, near, and after the flame tip $(x / d=30,40$, and 50$)$.
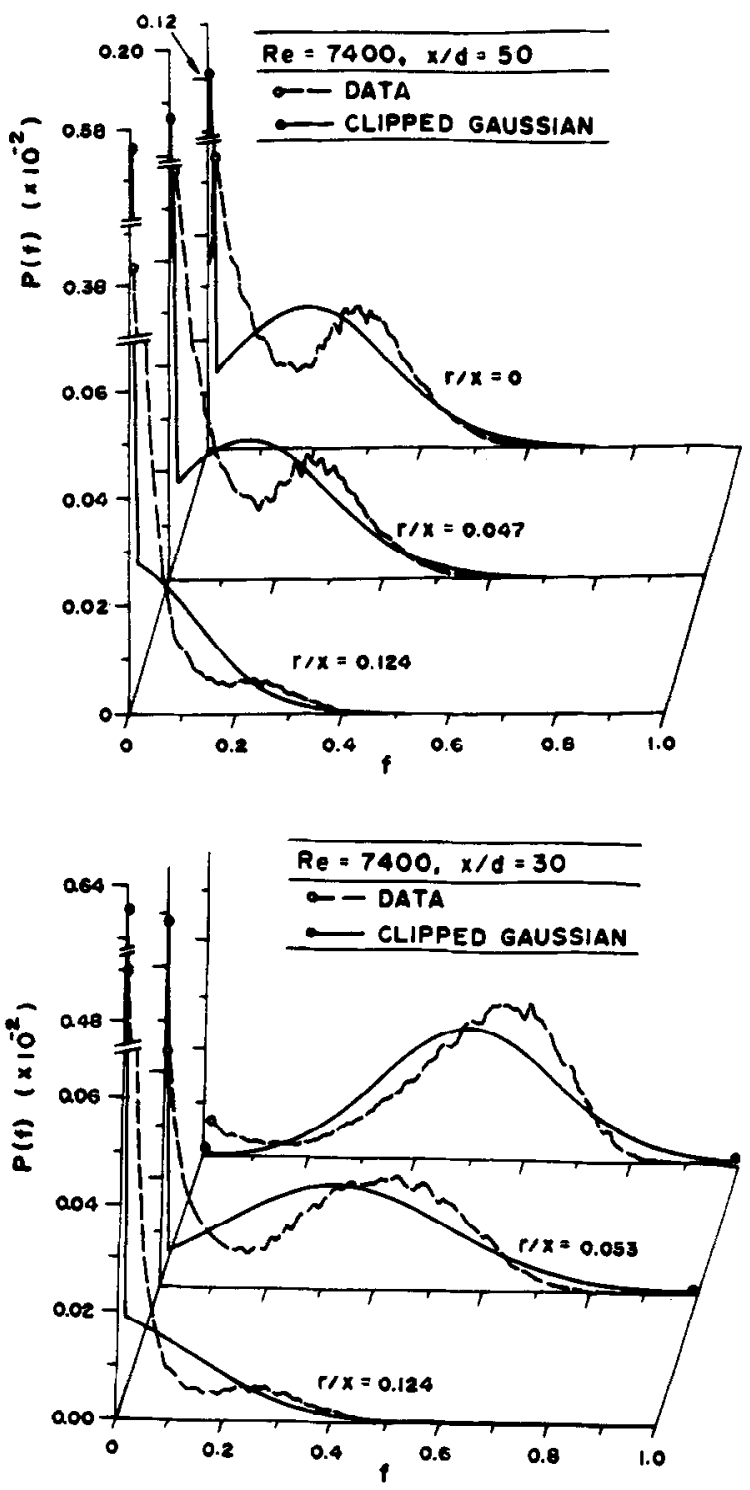

Fig. 2. Typical probability density functions of mixture fraction for a turbulent carbon monoxide/air diffusion flame. From Kounalakis and Faeth [30].

The correlations are plotted as a function of $\Delta r / \Gamma_{\mathrm{r}}$, where $\Delta r$ is the distance between the points and $\Gamma_{r}$ is the spatial integral scale in the radial direction. The spatial correlations exhibit remarkably little variation with either radial or axial position when plotted in this manner. A simple exponential 


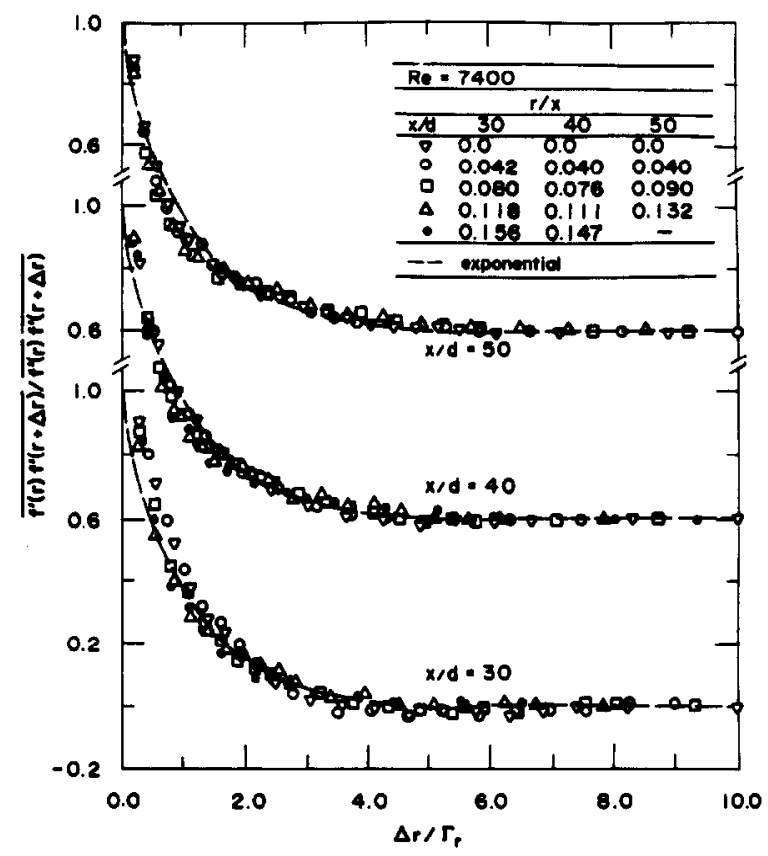

Fig. 3. Spatial correlations of mixture fraction fluctuations for a turbulent carbon monoxide/air diffusion flame. From Kounalakis and Faeth [30].

fit of the spatial correlation:

$$
\begin{aligned}
& \overline{f^{\prime}(r) f^{\prime}(r+\Delta r)} /{\overline{\left(f^{\prime 2}(r) f^{\prime 2}(r+\Delta r)\right)}}^{1 / 2} \\
& \quad=\exp \left(-\Delta r / \Gamma_{\mathrm{r}}\right)
\end{aligned}
$$

is also shown in the figure. The exponential function is seen to provide a reasonably good fit of the measurements, as illustrated in Fig. 3. This is partly due to experimental limitations, since the spatial resolution was not sufficient to resolve the smallest scales of the flow which are expected to modify the correlation near $\Delta r=0$ [30]. Nevertheless, the exponential expression provides a good representation of the larger scales that contain most of the signal energy and are expected to have the greatest influence on turbulence-radiation interactions. It should be noted, however, that these results differ from earlier findings in nearly constant density jets where radial correlations of mixture fraction fluctuations had the shape of a Frenkiel function [31,32] - these differences between combusting and noncombusting conditions must still be resolved.
Temporal correlations of mixture fraction fluctuations have been measured for the turbulent carbon monoxide jet diffusion flames as well [30]. These results were also relatively independent of position and could be correlated by an exponential function analogous to eq. (1) with time differences, $\Delta t$, normalized by the integral time scale, $\tau_{1}$ (subject to the same limitations as eq. (1) near $\Delta t=0)$. The exponential form of the low-resolution temporal correlation measurements agrees with earlier findings for noncombusting flows [32].

With exponential functions established as reasonable approximations of spatial and temporal correlations of mixture fraction fluctuations, the next problem is specification of integral scales. Measurements of these scales for turbulent carbon monoxide jet diffusion flames are illustrated in Fig. 4. The scales are normalized as $\Gamma_{\mathrm{r}} / x$ and as $\tau_{\mathrm{I}} u_{\mathrm{m}} /\left(x-x_{0}\right)$, where $u_{\mathrm{m}}$ is the average velocity at the burner exit and $x_{0}$ is a virtual origin at $x_{0} / d$ $=13$. When correlated in this manner, the measurements tend to collapse to single curves for a range of flame positions. The spatial integral scales are relatively independent of radial position and can be correlated as $\Gamma_{\mathrm{r}} / x=0.017$. In contrast, $\tau_{\mathrm{I}}$ is smallest at the axis. This behavior can be explained through Taylor's hypothesis, e.g., $\tau_{\mathrm{I}} \sim$ $\Gamma_{\mathrm{r}} / \bar{u}$, where $\bar{u}$ is the local time-averaged stream-

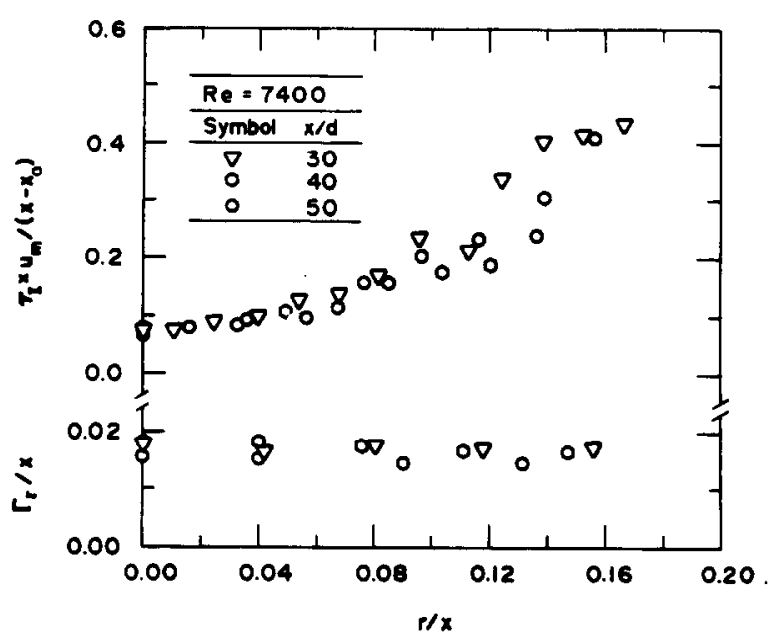

Fig. 4. Temporal and spatial integral scales in a turbulent carbon monoxide/air diffusion flame. From Kounalakis and Faeth [30]. 
wise velocity, while $\Gamma_{r}$ is nearly independent of radial position and $\bar{u}$ is a maximum at the axis.

Results concerning mixture fraction statistics in Figs. 2-4 were generally preserved as the Reynolds number of the carbon monoxide flames was increased [30]. Nevertheless, this only represents fragmentary findings for a single reactant combination, and generalization is needed to treat other flame systems. One proposal has been to assume that the radial spatial integral scale is proportional to the local dissipation length scale $[12,13]$, as follows:

$\Gamma_{\mathrm{r}}=C_{\mathrm{e}} C_{\mu}^{3 / 4} k^{3 / 2} / \epsilon$

where $C_{\mathrm{e}}$ is an empirical constant having a value in the range $5-7, C_{\mu}$ is a turbulence modeling constant having a value of 0.09 , and $k$ and $\epsilon$ are mass-weighted (Favre) averaged turbulence kinetic energy and dissipation found from structure predictions using a turbulence model. The temporal integral scale was then estimated using Taylor's hypothesis while assuming that streamwise and radial scales were the same, as follows:

$\tau_{\mathrm{I}}=\Gamma_{\mathrm{r}} / \tilde{u}$

where $\tilde{u}$ is the mass-weighted (Favre) averaged mean velocity in the streamwise direction. Eqs. (2) and (3) are consistent with the results illustrated in Fig. 4 but additional study of the approximations is certainly needed. For lack of an alternative, eqs. (2) and (3) will be used to find integral scales in the following.

\section{STOCHASTIC SIMULATION}

\section{Formulation}

The stochastic simulation provides realizations of mixture fraction distributions along radiation paths through the flow. Given the mixture fractions, the state relationships provide all other scalar properties so that spectral radiation intensities can be calculated from a narrow-band radiation model for each distribution. The resulting ensemble, or time series, of spectral radiation intensities is then used to compute moments, PDFs, correlations and power spectra of spectral radiation intensities in the usual manner. The formulation of the simulation and the narrow-band radiation model will be discussed in the following.

It will be assumed that the statistical properties of mixture fractions are known along the radiation path. This includes $P(f)$ (taken to be a clippedGaussian function), spatial correlations, and temporal correlations if temporal properties are needed (both taken to be exponential functions). Aside from isolated cases where measurements are available [30], these properties must be estimated from a model of the turbulent combustion process. For flows having relatively high Reynolds numbers, this is generally done using a turbulence model. Fortunately, for relatively simple flame geometries, like buoyant jet flames, turbulence models provide reasonably good estimates of scalar properties, including mean and fluctuating mixture fractions $[8-13,24,25]$. The necessary statistical properties of mixture fractions are then found as described earlier.

Due to the exponential form of the mixture fraction correlations, it is most convenient to carry out the simulation as an autoregressive process [15]. This involves finding the mixture fraction fluctuation at any point as a weighted sum of fluctuations at other points and a random shock. A procedure of this type encounters difficulties with any finite range PDF, since the fluctuation algorithm can easily generate a value of the variable which is beyond the range of the PDF. This is handled by transforming the simulation from $f$, which has a clipped-Gaussian PDF, to a corresponding Gaussian random variable $z$, with appropriate moments to match $P(f)$, so that

$f=z, 0 \leq z \leq 1 ; f=0, z<0 ; f=1, z>1$

Since the PDFs of $f$ and $z$ are not the same, correlations of $f$ and $z$ differ as well. Methods to find the appropriate correlations for $z$ will be taken up later.

Values of $z$ are simulated at a number of points along the radiation path. Following Box and Jenkins [15], the value of the fluctuation of $z$ at point $i, z_{i}^{\prime}$, is found as a weighted sum of fluctua- 
tions found earlier, $z_{j}^{\prime}$, where $j=i-1, \ldots, p$, and a random shock, $a_{i}$, as follows:

$z_{i}^{\prime}=\sum_{j=p}^{i-1} \phi_{i j} z_{j}^{\prime}+a_{i}^{\prime} ; \quad 1 \leq p \leq i-1$

The index $p$ is selected to eliminate points having small correlation coefficients with respect to point $i$. The $\phi_{i j}$ are weighting factors so that the simulation satisfies correlations between fluctuations at various points appearing in eq. (5). The parameter $a_{i}$ is an uncorrelated Gaussian random variable having a mean value of zero and a variance selected so that the simulation satisfies $P\left(z_{i}\right)$.

Box and Jenkins [15] derive expressions for the $\phi_{i j}$ and the variance of $a_{i}, \bar{a}_{i}^{2}$, as follows:

$\overline{z_{i}^{\prime} z_{k}^{\prime}}=\sum_{j=p}^{i-1} \phi_{i} \overline{z_{j}^{\prime} z_{k}^{\prime}} ; \quad k=p, \ldots, i-1$

$\bar{a}_{i}^{2}=\bar{z}_{i}^{\prime 2}-\sum_{j=p}^{i-1} \phi_{i j} \overline{z_{i}^{\prime} z_{j}^{\prime}}$

With the correlations between the various points known, eqs. (6) provide $i$-p linear equations, called the Yule-Walker equations, needed to find the $\phi_{i j}$. This system of equations has a symmetric positive definite matrix and can be solved readily using Cholesky factorization. Given the $\phi_{i j}, \bar{a}_{i}^{2}$ can be found since all quantities on the right-hand side of eq. (7) are known.

A time-independent simulation is initiated by making a random selection for point 1 , noting that $z_{i}^{\prime}=a_{1}$ from eq. (5) and $\bar{a}_{1}^{2}=\bar{z}_{1}^{\prime 2}$ from eq. (7). The regression relationships are then successively applied to find the remaining $z_{i}^{\prime}$ along the radiation path. Finally, the $f_{i}$ are found from eq. (4), noting that $z_{i}=\bar{z}_{i}+z_{i}^{\prime}$, followed by computation of spectral radiation intensities for this realization, as described earlier. This process is repeated a sufficient number of times to obtain statistically significant radiation properties.

The previously computed points in the regression process of eq. (5) only enter the calculations through their correlations; therefore, time-dependent simulations are essentially the same as timeindependent simulations after appropriately numbering points to keep track of them in space and time. This involves realizations of $f$ along the radiation path at times $\Delta t$ apart. The simulation is initiated by finding a realization using the time-independent solution. Realizations are then found at subsequent times considering correlations with all previous realizations, until temporal correlations are properly represented. Subsequently, the points at the earliest time are dropped when calculations for the next time are begun, for computational efficiency.

The main new difficulty with the time-dependent simulation is that two-point-two-time correlations are needed. Information of this type is not available; therefore, the following ad hoc approximation has been adopted for lack of an alternative [13]:

$\overline{z_{i}^{\prime}(t) z_{j}^{\prime}(t-k \Delta t)}=R_{i}(k \Delta t) \overline{z_{i}^{\prime} z_{j}^{\prime}}$

where $R_{i}(k \Delta t)$ is the temporal correlation coefficient of $z_{i}$ fluctuations at a time delay of $k \Delta t$. Naturally, it would be just as plausible to use $R_{j}(k \Delta t) \overline{z_{i}^{\prime} z_{j}^{\prime}}$ on the right-hand side of eq. (8) for a stationary turbulent flow. The differences between these possibilities provides a measure of potential errors resulting from the use of eq. (8). Since $\Gamma_{r}$ is nearly constant over a cross-section of the flow, eq. (3) indicates that errors are greatest in regions where $\tilde{u}$ varies rapidly. Fortunately, spatial correlations become small for separation distances of $\Gamma_{\mathrm{r}}$ and $\tilde{u}$ does not vary significantly over such distances, providing some justification for the approximation.

When temporal correlations are exponential, use of eq. (8) for two-point-two-time correlations leads to substantial simplification of time-dependent simulations. Carrying out a derivation similar to that of Box and Jenkins [15] for a pure time series with stationary statistics and an exponential temporal correlation yields similar results for the combined spatial/temporal simulation with temporal correlations varying according to eq. (8), namely the $\phi_{i j}=0$ for all points at times less than $t-\Delta t$. Thus, only the realization at $t-\Delta t$ must be retained while developing the realization at $t$, vastly reducing the storage and computational requirements of the simulation.

Another useful simplification is that radiation predictions are relatively insensitive to the func- 
tional form of the spatial correlation, since they are found by integrating properties along a radiation path $[13,33]$. Thus, temporal simulations using statistically independent points spaced a distance $\Gamma_{r}$ apart along the radiation path yielded results that were essentially the same as simulations that satisfied twenty-point fits of spatial correlations along the radiation paths [13]. This simplification reduces the simulation to a first-order (Markov) process in time at each point, for an exponential temporal correlation, yielding [15]:

$z_{i}^{\prime}(t)=R_{i}(\Delta t) z_{i}^{\prime}(t-\Delta t)+a_{i}$

where

$\overline{a_{i}^{2}}=\left(1-R_{i}(\Delta t)^{2}\right){\overline{z_{i}^{\prime}}}^{2}$

\section{Correlation corrections}

Initial time-series simulations of mixture fraction distributions involved the approximation that correlations of $f$ and $z$ were the same $[12,13]$. This was adequate in most regions of the flames but discrepancies between actual and simulated correlations of mixture fraction fluctuations were significant in regions where either air or fuel intermittencies were high [13]. The cause of the difficulty is the transformation from $f$ to $z$, since $z$ has an infinite range while $0 \leq f \leq 1$. This implies that the correlations of the fluctuations of $z$ must be corrected in order to properly simulate the correlations of the fluctuations of $f$.

A generalized correction of the $z$ correlations has been developed for any two points, $i>j$, having identical mean and fluctuating mixture fractions, $\bar{f}_{i}=\bar{f}_{j}=f$ and $\overline{f_{i}^{\prime 2}}=\overline{f_{j}^{\prime 2}}=\overline{f^{2}}$, i.e., for temporal correlations at stationary conditions. The simulation is carried out with the $z$ variable where $\bar{z}_{i}=\overline{z_{j}}=\bar{z}$ and $\overline{z_{i}^{\prime 2}}=\overline{z_{j}^{\prime 2}}=\overline{z^{\prime 2}}$ can be found from the transformation of eq. (4). In order for the simulation to yield the correct correlation, $\overline{f_{i}^{\prime} f_{j}^{\prime}}$, the value of $\overline{z_{i}^{\prime} z_{j}^{\prime}}$ must be corrected so that the following equation is satisfied

$$
\begin{aligned}
& \overline{f_{i}^{\prime} f_{j}^{\prime}}+\overline{f^{2}} \\
& \quad=\int_{-\infty}^{\infty} f\left(z_{i}\right) P\left(z_{i}\right) \int_{-\infty}^{\infty} f\left(z_{j}\right) P\left(z_{j}: z_{i}\right) \mathrm{d} z_{j} \mathrm{~d} z_{i}
\end{aligned}
$$

where $f\left(z_{i}\right)$ and $f\left(z_{j}\right)$ are obtained from eq. (4) and $P\left(z_{j}: z_{i}\right)$ is the probability density function of $z_{j}$ given $z_{i}$. Now, the correct correlation for the $z$ variables can be found by considering an autoregressive process between the two points under the present approximations, as follows:

$z_{j}^{\prime}=z_{i}^{\prime}\left(\overline{z_{i}^{\prime} z_{j}} / \overline{z^{\prime 2}}\right)+a_{j}$

where $a_{j}$ has a Gaussian PDF with

$\overline{a_{j}^{2}}={\overline{z^{\prime}}}^{2}-\left(\overline{z_{i}^{\prime} z_{j}^{\prime}}\right)^{2} /{\overline{z^{\prime}}}^{2}$

Then, for any realization of $z_{i}^{\prime}, P\left(z_{j}: z_{i}\right)$ is a Gaussian distribution having a mean value of $\bar{z}+$ $\left.z_{i}^{\prime} \overline{z_{i}^{\prime} z_{j}^{\prime} / z^{\prime 2}}\right)$ and a variance of $\bar{a}_{j}^{2}$, while $P\left(z_{i}\right)$ is a Gaussian distribution having a mean value of $\bar{z}$ and a variance of $\overline{z^{\prime}}$. Substituting these expressions, along with $f\left(z_{i}\right)$ and $f\left(z_{j}\right)$ from eq. (4) into eq. (11) yields an expression relating $\overline{f_{i}^{\prime} f_{j}^{\prime}}$ and $\overline{z_{i}^{\prime} z_{j}^{\prime}}$. This expression must be evaluated numerically for a clipped-Gaussian $P(f)$. The procedure was to select values of $\bar{z}, \overline{z^{\prime}}$ and $\overline{z_{i}^{\prime} z_{j}^{\prime}}$ and then find the corresponding values of $\bar{f}, \overline{f^{\prime}}{ }^{2}$, and $\overline{f_{i}^{\prime} f_{j}^{\prime}}$. Present results were found by integrating over the region within 5 standard deviations from the mean of the PDFs.

Since the temporal correlations of $f$ are exponential, it was convenient to fit the correlations of $z$ in the same manner and to express the corrections of the correlations as ratios between the temporal integral scales of $f$ and $z, \tau_{f} / \tau_{z}$. This ratio is plotted in Fig. 5 as a function of $\left(\bar{f}^{\prime 2}\right)^{1 / 2}$ with $\bar{f}$ as a parameter. The results are symmetric with respect to $\bar{f}=0.5$. The plots of $\tau_{f} / \tau_{z}$ at a particular value of $f$ are terminated at the maximum possible value of $\left(\bar{f}^{\prime 2}\right)^{1 / 2}$, i.e., where $P(f)$ degenerates to Dirac delta functions at $f=0$ and 1. The ratio of $\tau_{f} / \tau_{z}$ decreases from unity as $\left(\bar{f}^{\prime}\right)^{1 / 2}$ increases and $\bar{f}$ approaches either 0 or 1 . Thus, there is no correction when $z$ remains in the range $0-1$ where $z=f$. Whenever $z<0$ or $z>1$, however, $z^{2}>f^{2}$ and the correlation for $f$ generally is less than the correlation for $z$ so that $\tau_{f} / \tau_{z}$ is less than unity.

Simulations using corrected correlations for $z$ were evaluated for $\overline{z_{i}^{\prime} z_{j}^{\prime} / z^{\prime 2}}>0.1$. Using $10^{4}$ reali- 


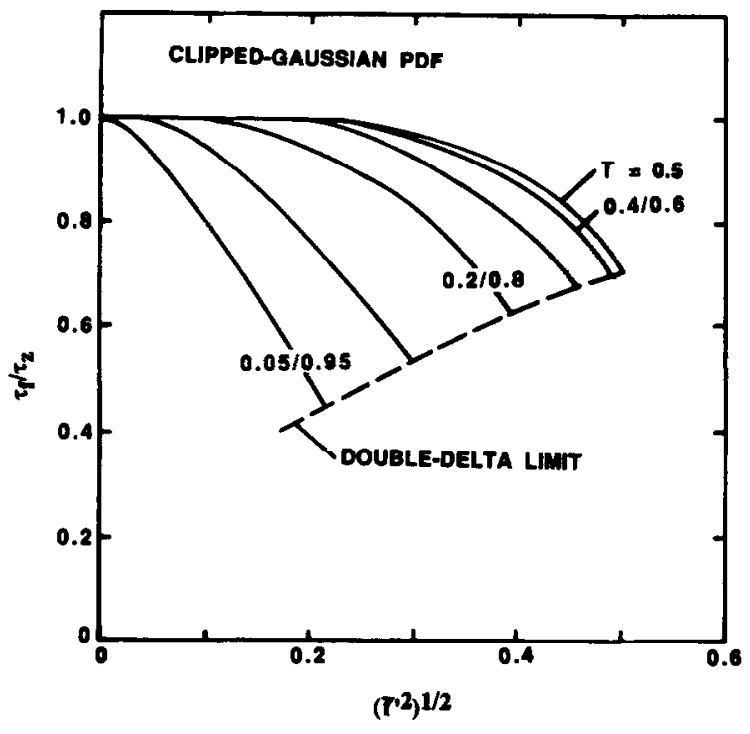

Fig. 5. Ratio of simulated and original integral scales for exponential correlations of functions having clipped-Gaussian probability density functions.

zations, values of $\bar{f}$ and $\bar{f}^{2}$ were satisfied within $1 \%$ while values of $\overline{f_{i}^{\prime} f_{j}^{\prime} / f^{\prime 2}}$ were satisfied within 3\%. Analogous calculations to find the corresponding corrections of the correlations when $\bar{f}$ and $\bar{f}^{2}$ are not the same at the two points are straightforward on a case-by-case basis.

\section{Narrow-band radiation model}

Given the distribution of scalar properties along a radiation path, through the stochastic simulation of mixture fractions and the state relationships, spectral radiation intensities are found by solving the equation of radiative transfer along the path. Present results involved using a narrow-band model, ignoring scattering, due to Ludwig et al. [34]. The procedure uses the Goody statistical narrow-band model, with the Curtiss-Godson approximation to account for absorption along inhomogeneous gas paths. This model accounts for the infrared gas bands of water vapor, carbon dioxide, carbon monoxide, and methane, as well as continuum radiation from soot. Radiation contributions of other species in hydrogen, carbon monoxide, and hydrocarbon flames burning in air are generally negligible since these species have small concentrations in regions where temperatures are high.

\section{RESULTS AND DISCUSSION}

Some comparisons between simulated and measured radiation properties will be considered in order to illustrate the nature and effectiveness of the simulations. The discussion will be limited to results reported by Kounalakis et al. [12,13] for vertical turbulent hydrogen and carbon monoxide jet flames burning in still air. Spectral radiation intensities, $i_{\lambda}$, were measured for horizontal radiation paths through the axis of the flames. Predictions were based on the present formulation of the stochastic simulation of mixture fraction distributions. As noted earlier, twenty-point fits of spatial correlations in the simulation yielded essentially the same results as the simplified formulations of eqs. (9) and (10); therefore, the following results are based on the simplified formulation. Mixture fraction statistics were estimated based on structure predictions using a turbulence model. This introduces uncertainties although the turbulence model yielded reasonably good predictions of scalar structure for the same flames during earlier studies $[8,9]$.

Predicted and measured probability density functions of $i_{\lambda}$ are illustrated in Fig. 6 for positions before, near, and after the tip of a hydrogen jet flame $(x / d=50,90$, and 130). These results are for a wavelength $\lambda=2520 \mathrm{~nm}$ which is within a prominent infrared gas radiation band for water vapor. Near the burner, the PDFs are relatively symmetric but they become increasingly skewed as distance from the burner exit increases. This is an effect of air intermittency as mean radiation levels become small, since the spectral intensity can never be negative while the mean value is generated by occasional periods of high radiation levels. The stochastic predictions represent the measurements reasonably well, particularly for the small path diameter which more closely approximates the negligible path diameter of the simulation.

Predicted and measured temporal power spectra of spectral radiation intensities, $E_{\lambda}(n)$, are illustrated in Fig. 7 for positions before, near, and 


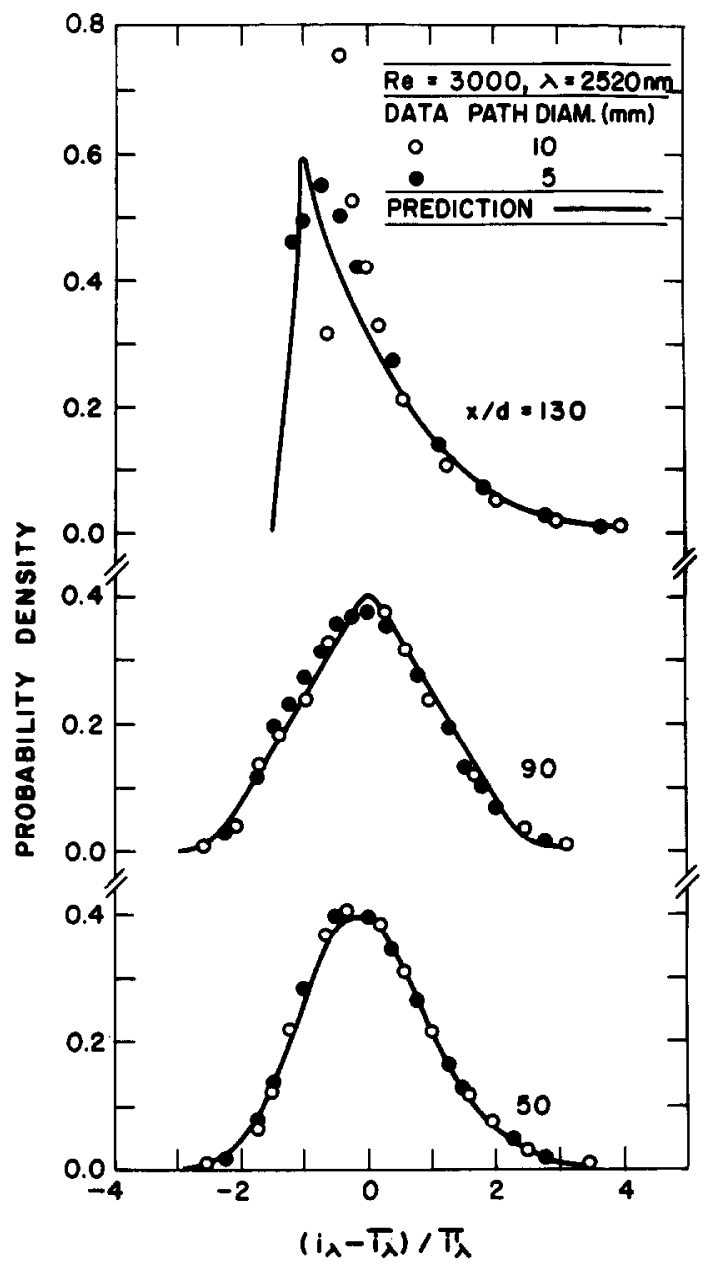

Fig. 6. Measured and predicted probability density functions of spectral radiation intensities for a turbulent hydrogen/air diffusion flame. From Kounalakis et al. [12].

after the tip of a carbon monoxide jet flame $(x / d=35,50$, and 65$)$. The power spectra are plotted as a function of frequency, $n$, both normalized by the characteristic frequency, $\bar{u}_{c} / x$, where $\bar{u}_{c}$ is the mean velocity at the flame axis. The spectra exhibit a break frequency with an energy-containing region having a nearly constant $E_{\lambda}(n)$ at low frequencies, followed by decay of $E_{\lambda}(n)$ with increasing frequency beyond the break frequency. Normalized break frequencies increase somewhat with increasing distance from the burner. This follows since the high temperature region that contributes most to radiant emission is located off axis near the burner and moves toward the axis with increasing distance above the burner. Since temporal integral scales are smallest near the axis (see Fig. 4), this implies a corresponding increase in the break frequency when normalized by properties at the axis.

The predictions provide reasonable estimates of break frequencies and signal properties in the energy-containing region for the results illustrated in Fig. 7. The main deficiency of the predictions is that they underestimate the rate of decay of $E_{\lambda}(n)$ at high frequencies. Two main reasons can be advanced for this behavior. First of all, spectral intensities were measured for a finite diameter radiation path. This tends to average out highfrequency effects over the cross-section of the radiation path in comparison to predictions which represent an infinitely thin path. An indication of this effect can be seen by comparing measurements for 5- and 10-mm-diameter paths appearing in Fig. 7, which show that the spectra decay more rapidly for the larger-diameter path. Secondly, the

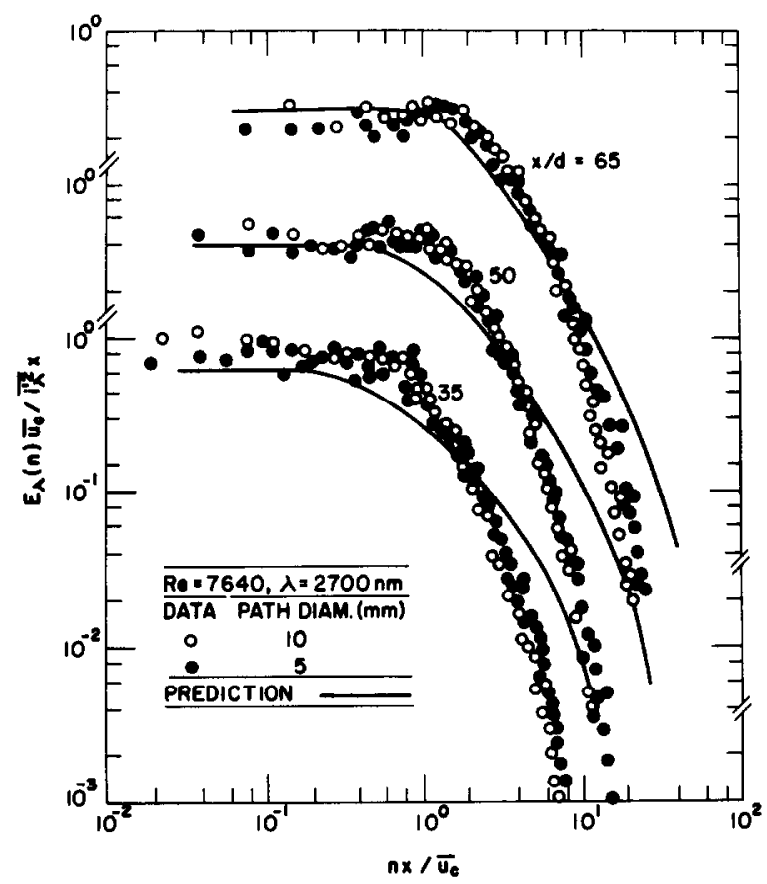

Fig. 7. Measured and predicted temporal power spectral densities of spectral radiation intensities for a turbulent carbon monoxide/air diffusion flame. From Kounalakis et al. [13]. 
exponential correlation function used in the stochastic simulation does not properly truncate high-frequency fluctuations as turbulent microscales are approached, as noted earlier. This causes the predictions to overestimate high frequency signal levels. Resolving these problems would require extension of the stochastic simulation, to allow simulation of groups of parallel radiation paths so that they can be summed over a finite-diameter path and to accommodate high-frequency cut-offs associated with turbulence microscales when simulating correlations. However, since spectral intensity signal energies are relatively small when the discrepancy becomes significant, such extensions are not needed for most applications.

\section{CONCLUSIONS}

The use of statistical time-series techniques to treat nonlinear interactions during turbulent combustion processes was described. Turbulenceradiation interactions were used to illustrate the method; however, other turbulence interaction problems for combusting flows require a similar treatment of scalar properties. Existing evidence suggests that scalar properties are strongly correlated through state relationships in turbulent diffusion flames and can be simulated by only simulating a conserved-scalar like mixture fraction. The statistics of mixture fractions in turbulent diffusion flames can be approximated by a clippedGaussian PDF and exponential spatial and temporal correlations, at least for the large-scale features that dominate radiation properties. Stochastic simulations using statistical time-series techniques must be modified to account for the finiterange PDF of mixture fraction. This involved transformation to a new variable having a Gaussian PDF and finding appropriate corrections for the correlations in terms of the new variable. An autoregressive process that reproduced the PDFs and spatial and temporal correlations of mixture fractions yielded an effective simulation to find the statistical properties of spectral radiation intensities from turbulent jet diffusion flames. Thus, additional development and application of the method appears to be warranted.

\section{ACKNOWLEDGEMENT}

This research was supported by the Center for Fire Research of the National Institute of Standards and Technology (formerly the National Bureau of Standards), Grant No. 60NANB8D0833 with H.R. Baum serving as Scientific Officer.

\section{REFERENCES}

1 J.-S. Shuen, A.S.P. Solomon, Q.-F. Zhang and G.M. Faeth, Structure of particle-laden jets: measurements and predictions, American Institute of Aeronautics and Astronautics Journal, 23 (1985) 396-404.

2 T.-Y. Sun and G.M. Faeth, Structurc of turbulent bubbly jets, International Journal of Multiphase Flow, 12 (1986) 99-126.

3 A. Picart, A. Berlemont and G. Gouesbet, Modeling and predicting turbulence fields and dispersion of discrete particles transported by turbulent flows, International Journal of Multiphase Flow, 12 (1986) 237-261.

4 M.R. Maxey, The gravitational settling of aerosol particles in homogeneous turbulence and random flow fields, Journal of Fluid Mechanics, 174 (1987) 441-465.

5 R.N. Parthasarathy and G.M. Faeth, Turbulent dispersion of particles in self-generated homogeneous turbulence, Journal of Fluid Mechanics, in press.

6 A.S.P. Solomon, J.-S. Shuen, Q.-F. Zhang and G.M. Faeth, Measurements and predictions of the structure of evaporating sprays, Journal of Heat Transfer, 107 (1985) 679-686.

7 J.S. Shuen, A.S.P. Solomon and G.M. Faeth, Drop-turbulence interactions in a diffusion flame, American Institute of Aeronautics and Astronautics Journal, 24 (1986) 101-108.

8 J.P. Gore, S.-M. Jeng and G.M. Faeth, Spectral and total radiation properties of turbulent carbon monoxide/air diffusion flames, American Institute of Aeronautics and Astronautics Journal, 25 (1987) 339-345

9 J.P. Gore, S.-M. Jeng and G.M. Faeth, Spectral and total radiation properties of turbulent hydrogen/air diffusion flames, Journal of Heat Transfer, 109 (1987) 165-171.

10 J.P. Gore and G.M. Faeth, Structure and spectral radiation properties of turbulent ethylene/air diffusion flames, Twenty-First Symposium (International) on Combustion, The Combustion Institute, Pittsburgh, PA, 1986, pp. 1521-1531.

11 J.P. Gore and G.M. Faeth, Structure and radiation properties of luminous turbulent acetylene/air diffusion flames, Journal of Heat Transfer, 110 (1988) 173-181.

12 M.E. Kounalakis, J.P. Gore and G.M. Faeth, Turbulence/ radiation interactions in nonpremixed hydrogen/air flames, Twenty-Second Symposium (International) on Combustion, The Combustion Institute, Pittsburgh, PA, 1988, pp. 12811290.

13 M.E. Kounalakis, J.P. Gore and G.M. Faeth, Mean and fluctuating radiation properties of turbulent nonpremixed 
carbon monoxide/air flames, Journal of Heat Transfer, 111 (1989) 1021-1030.

14 R.H. Kraichnan, Diffusion by a random velocity field, Physics of Fluids, 13 (1970) 22-31.

15 G.E.P. Box and G.M. Jenkins, Time Series Analysis, Holden Day, San Francisco, CA, revised edition, 1976, pp. 47-84.

16 S.P. Burke and T.E.W. Schumann, Diffusion flames, Industrial and Engineering Chemistry, 20 (1928) 998-1004.

17 R.W. Bilger, Turbulent jet diffusion flames, Progress in Energy and Combustion Science, 1 (1976) 87-109.

18 R.W. Bilger, Reaction rates in diffusion flames, Combustion and Flame, 30 (1977) 277-284.

19 S. Gordon and B.J. McBride, Computer Program for Calculation of Complex Chemical Equilibrium Compositions, Rocket Performance, Incident and Reflected Shocks, and Chapman-Jouguet Detonations, NASA SP-273, Washington, DC, 1971.

20 K.C. Smyth, J.H. Miller, R.C. Dorfman, W.G. Mallard and R.J. Santoro, Soot inception in a methane/air diffusion flame as characterized by detailed species profiles, Combustion and Flame, 62 (1985) 157-181.

21 K. Saito, F.A. Williams and A.S. Gordon, Structure of laminar coflow methane-air diffusion flames. Journal of Heat Transfer, 108 (1986) 640-648.

22 Y.R. Sivathanu and G.M. Faeth, Generalized state relationships for scalar properties in nonpremixed hydrocarbon/air flames, Combustion and Flame, in press.

23 J.H. Kent and F.A. Williams, Extinction of laminar diffusion flames for liquid fuels, Fifteenth Symposium (International) on Combustion, The Combustion Institute, Pittsburgh, PA, 1974, pp. 315-325.

24 S.-M. Jeng and G.M. Faeth, Species concentrations and turbulence properties in buoyant methane diffusion flames, Journal of Heat Transfer, 106 (1984) 721-727.
25 Y.R. Sivathanu, J.P. Gore and G.M. Faeth, Scalar properties in the overfire region of sooting turbulent diffusion flames, Combustion and Flame, 73 (1988) 315-329.

26 Y.R. Sivathanu and G.M. Faeth, Soot volume fractions in the overfire region of turbulent diffusion flames, Combustion and Flame, 81 (1990) 133-149.

27 Y.R. Sivathanu and G.M. Faeth, Temperature/soot volume fraction correlations in the fuel rich region of buoyant turbulent diffusion flames, Combustion and Flame, 81 (1990) $150-165$.

28 F.C. Lockwood and A.S. Naguib, The prediction of the fluctuations in the properties of free, round-jet, turbulent diffusion flames, Combustion and Flame, 24 (1975) 109-124.

29 M.-C. Lai and G.M. Faeth, Turbulence structure of vertical adiabatic wall plumes, Journal of Heat Transfer, 109 (1987) 663-670.

30 M.E. Kounalakis and G.M. Faeth, Measurement of mixture-fraction correlations in turbulent jet diffusion flames, Proceedings of Fall Technical Meeting, Eastern Section of the Combustion Institute, Pittsburgh, PA, 1989.

31 S. Corrsin and M.S. Uberoi, Spectra and Diffusion in $a$ Round Turbulent Jet, NACA Report No. 1040, Washington, DC, 1951.

32 H.A. Becker, H.C. Hottel and G.C. Williams, The nozzlefluid concentration field of the round, turbulent, free jet, Journal of Fluid Mechanics, 30 (1967) 285-303.

33 W.L. Grosshandler and P. Joulain, The effect of large-scale fluctuations on flame radiation, Progress in Astronautics and Aeronautics, 105(II) (1986) 123-152.

34 C.B. Ludwig, W. Malkmus, J.E. Reardon and J.A. Thomson, Handbook of Infrared Radiation from Combustion Gases, NASA SP-3080, Washington, DC, 1973. 